\title{
The epidemiology of postpartum malaria: a systematic review
}

\author{
Machteld E Boel ${ }^{1,2^{*}}$, Marcus J Rijken ${ }^{1}$, Bernard J Brabin ${ }^{2,3}$, François Nosten ${ }^{1,4,5}$ and Rose McGready ${ }^{1,4,5}$
}

\begin{abstract}
Pregnant women are more susceptible to malaria than their non-pregnant counterparts. Less is known about the risk of malaria in the postpartum period. The epidemiology of postpartum malaria was systematically reviewed. Eleven articles fitted the inclusion criteria. Of the 10 studies that compared malaria data from the postpartum period with pregnancy data, nine studies suggested that the risk for malaria infection decreased after delivery. All three studies that compared postpartum data with non-pregnant non-postpartum women concluded that the risk did not return to pre-pregnancy levels immediately after delivery. The results of this review have to be carefully interpreted, as the majority of studies were not designed to study postpartum malaria, and there was large variability in study designs and reported outcomes. Current evidence suggests an effort should be made to detect and radically cure malaria during pregnancy so that women do not enter the postpartum period with residual parasites.
\end{abstract}

Keywords: Malaria, Postpartum, Pregnancy, Delivery

\section{Background}

Pregnant women are more susceptible to malaria than their non-pregnant counterparts. This was described nearly a century ago[1], and applies to all malaria regions in the world, regardless of transmission intensity $[2,3]$. Primigravida tend to be more susceptible than multigravida, as women gain immunity against malaria during successive pregnancies, particularly in areas of high transmission[4].

To control Plasmodium falciparum malaria during pregnancy, the WHO Roll Back Malaria recommends several strategies: personal protection with insecticide impregnated bed nets (ITNs), intermittent preventive treatment (IPTp) and case management of anaemia and malaria illness with effective anti-malarial drugs[5]. A much less emphasized part of this recommendation is that women in the postpartum period should be encouraged to use ITNs. There are no other recommended preventive strategies for postpartum women. However, malaria was one of the leading causes of hospital admission and maternal death among postpartum women in Zambia[6] and India[7]. Little is known about the

\footnotetext{
* Correspondence: machteld@shoklo-unit.com

'Shoklo Malaria Research Unit, PO Box 46, 63110, Mae Sot Tak, Thailand Full list of author information is available at the end of the article
}

epidemiology and pathophysiology of postpartum malaria. In theory, the increased susceptibility during pregnancy normalizes immediately after delivery when the placenta, where the parasites can adhere during pregnancy, is expelled. This is supported by the reports of spontaneous clearance of $P$. falciparum parasites within 24 hours after delivery in high transmission areas in Africa[8,9]. In contrast, in Malawi[10] among women of which some were screened at day 0 , others at day 1 , 2 , until 1 week after delivery, the proportion with a positive malaria blood smear was constant each day over the first seven days (K. Msyamboza, personal communication). This suggests that other factors may play a role, such as behavioural and physiological changes that attract mosquitoes during pregnancy[11], suppression of cell-mediated immunity and changes in maternal hormonal levels. These factors take much longer to normalize[12,13], and might extend beyond the usual limit of the postpartum period which is defined by $\mathrm{WHO}$ as the period until six weeks after delivery[14]. The primary objective of this systematic review was to describe the epidemiology of malaria in the postpartum period. This was achieved by comparing postpartum data: 1) to longitudinally collected malaria data from the same women during pregnancy; 2) to malaria data from non-pregnant,
C Biomed Central

C 2012 Boel et al; licensee BioMed Central Ltd. This is an Open Access article distributed under the terms of the Creative Commons Attribution License (http://creativecommons.org/licenses/by/2.0), which permits unrestricted use, distribution, and reproduction in any medium, provided the original work is properly cited. 
non-postpartum control women matched for age, area of residence and time; or 3) to both 1 and 2 .

\section{Methods}

References for this review were identified through searches of MEDLINE and the Malaria in Pregnancy library[15] with the terms 'malaria' AND 'postpartum' OR 'puerperium' using a combination of $\mathrm{MeSH}$ headings and keywords. The search was limited to humans, clinical trials, in English and Spanish language until 28 February 2011. Two authors independently performed eligibility assessment and, if disagreements were not resolved by consensus, a decision was made by a third author. The reporting guidance as described in the PRISMA statement was closely followed [16].

Criteria for inclusion were: a) laboratory confirmation of malaria in the postpartum period; b) clarity about the timing of malaria sampling; and c) similarity in epidemiological indicators to describe malaria in pregnant, postpartum and control women. Exclusion criteria were: a) selection bias e.g. only women with fever, b) seasonal bias e.g. longitudinal cohort study with enrolment only during one season in an area with seasonal transmission, c) unavailability of locally comparable data (pregnant or general population during the same time period) and d) less than two weeks follow up after delivery. If crucial data were missing, the authors were approached to provide additional information. Information extracted from each study included: (1) characteristics of the study (study subject, time period, location, enrolment criteria, length and frequency of follow up during pregnancy and postpartum, passive or active case detection, malaria species, parasite densities, symptoms, methods of diagnosing malaria, malaria endemicity and treatment) (2) quality of measures that could affect postpartum susceptibility (type of study, use of IPTp or chemoprophylaxis, samples taken during pregnancy or delivery, drug efficacy, treatment criteria, self-treatment with antimalarials, use of control patients and percentage of lost to follow up) and (3) outcome measures (incidence, prevalence or rate ratios of malaria infection during pregnancy and postpartum and in non-pregnant non postpartum controls if available). To allow comparisons between different studies the outcome measures of each study were recalculated into proportions of infected women at certain time points during pregnancy, postpartum or in control women with confidence limits and p-values. In studies where all women were given chemoprophylaxis or IPTp, only the malaria data of the first antenatal visit were used for comparison with postpartum data. When IPTp or chemoprophylaxis was used in a randomized controlled trial, data of the placebo arm and the study drug arm were analysed separately.

\section{Results}

The literature search provided a total of 162 abstracts of which 128 did not meet the inclusion criteria (Figure 1). The full text of the remaining 34 citations was examined in detail and of these, eleven studies met the inclusion criteria[17-27] (Figure 1). Two of these articles described the same population but used different methodology to detect malaria[23,25].

\section{Characteristics of the included studies}

The eleven included studies were conducted between 1968 and 2005. Only three studies were designed to measure postpartum malaria[19,24,25]. The central research question of the eight remaining studies pertained to pregnancy related subjects (Additional file 1: Table S1), but the study follow-up was extended into the postpartum period for at least two months. All studies were conducted in highly endemic malarious areas: 10 studies were from Africa, where P. falciparum is the main specie of malaria and one in Papua New Guinea (PNG) where Plasmodium vivax is co-endemic with $P$. falciparum and Plasmodium malariae. These species were included in the analysis of the paper from PNG [17], but the analysis in the papers from Africa was restricted to P.falciparum, although three African studies mentioned P. malariae and/or Plasmodium ovale to occur occasionally[22,24,26].

\section{Quality measures of the included studies}

In 10 of the 11 studies postpartum malaria was compared with longitudinal data from women during pregnancy or at delivery (Additional file 2: Table S2). Three of these studies compared postpartum data with nonpregnant, non-postpartum women as well $[17,19,22]$, whereas one study only used the latter as a control

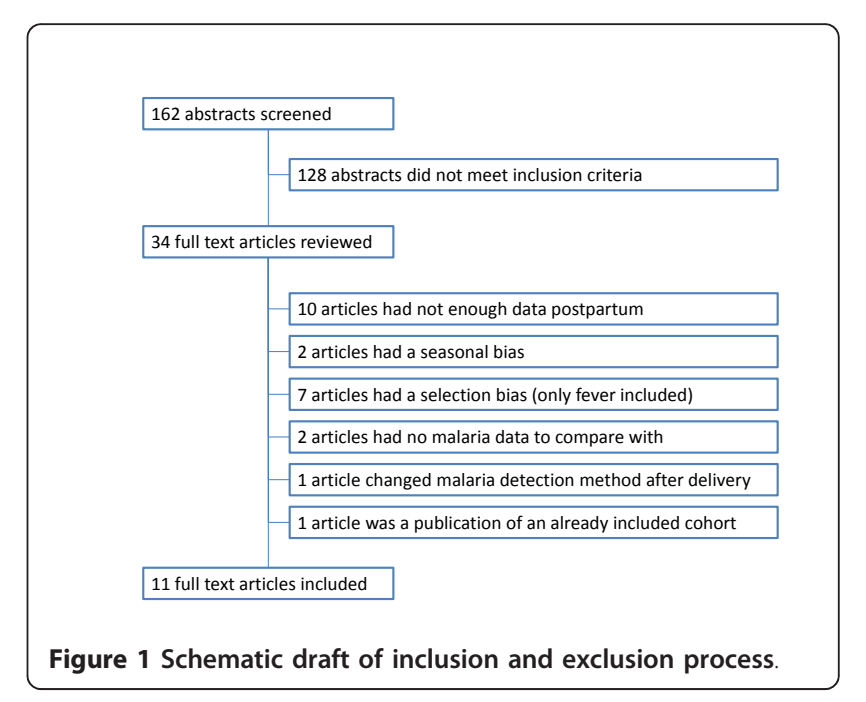


group[24]. Figure 2 shows the active screening regimen of each study.

The methods to describe post partum malaria varied in epidemiological indicators between studies. Distinction between primi- or multigravida was made in seven studies (Additional file 2: Table S2). Two studies conducted P.falciparum genotyping on delivery samples and postpartum samples[24,25], and sensitive detection for submicroscopic infection at delivery and postpartum was performed in one study[25].

Chloroquine or sulphadoxine-pyrimethamine were used for treatment, chemoprophylaxis or IPTp, while resistance of P.falciparum parasites to these drugs was reported in six of these papers (Additional file 2: Table S2). In four of them, definitions about drug resistance were available, either in the text or by referring to other articles $[17,23,25,26]$. Self-treatment with anti-malarials was described in two studies[19,22]. Various treatment protocols of malaria episodes in pregnancy were used: in four studies any detected parasitaemia was treated regardless of symptoms, whereas in four other studies only women with symptomatic infections or high parasite counts were treated with anti-malarials (Additional file 2: Table S2).

\section{Main outcome of included studies}

The pertinent points relating to postpartum susceptibility from each of the 11 manuscripts are discussed in detail in Additional file 3. Nine of the 10 included studies that compared malaria during the postpartum period with malaria during pregnancy, showed a decline in the proportions of malaria positive women during the postpartum period (Figure 3, Additional file 3). The three studies that compared postpartum data with nonpregnant, non-postpartum controls found higher proportions in postpartum women compared to controls

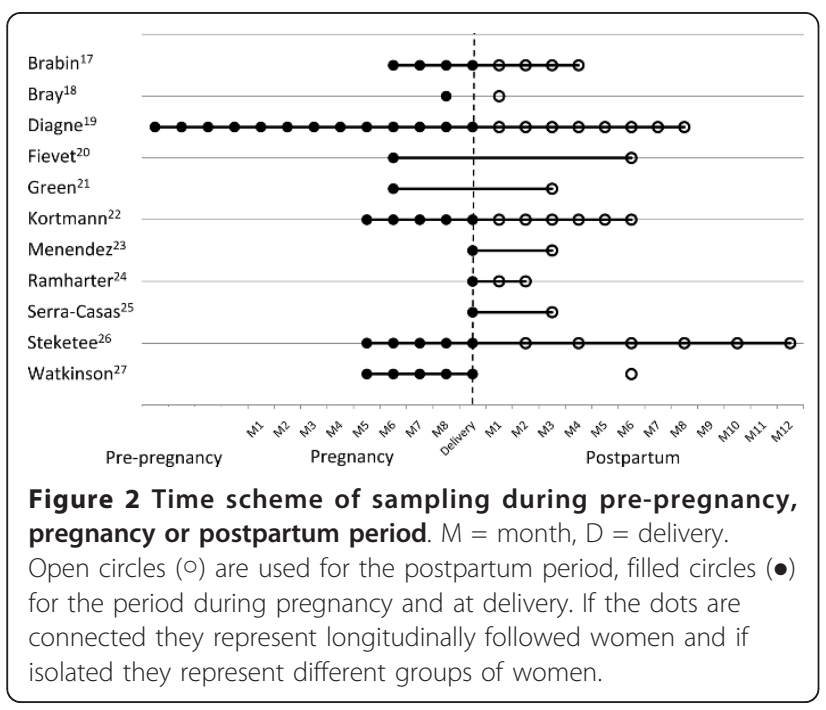

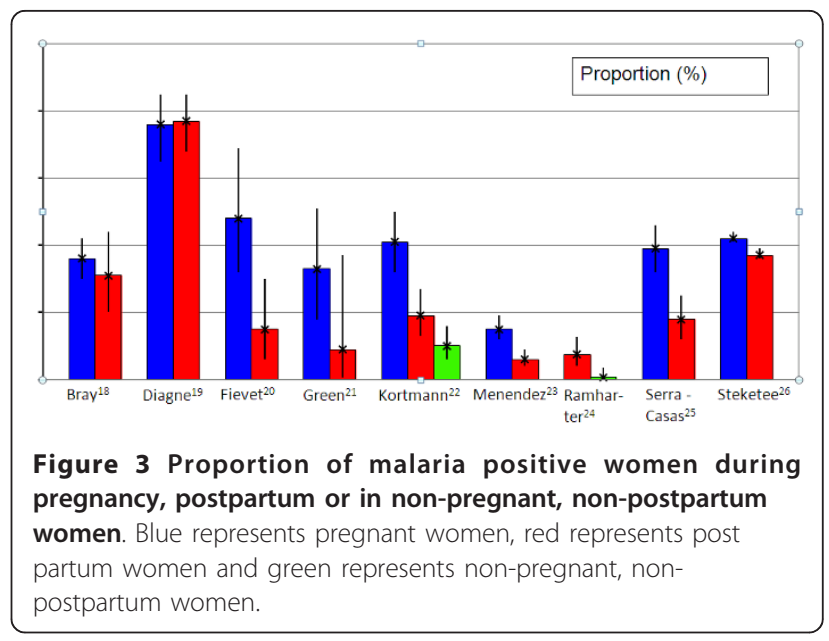

$[19,22,24]$. Of the seven studies that analysed malaria in the postpartum period separately for different gravidities, six studies observed more malaria episodes in primiparous postpartum women compared to multiparous women (RR calculated from 3 studies with proportions available $[23,25,27]=2.77$ (95\% CI $1.61-4.77), \mathrm{p}<$ $0.001)$. Only the study from Gabon showed a non-significant difference in risk for primiparous compared to multiparous postpartum women $(\mathrm{RR}=0.3$ (95\% CI 0.0 1.4), $\mathrm{p}=0.12$.)

Main outcome of studies witht IPTp or chemoprophylaxis In two of the three studies, where weekly chloroquine chemoprophylaxis was used during pregnancy and stopped at delivery, an increase in malaria after delivery was demonstrated. In PNG [17], the P. falciparum and $P$. vivax incidence per person-month increased until four months postpartum for all gravidae compared to pregnancy, and this was significant ( $\mathrm{p}=0.002)$ for multiparous women with $P$. vivax. Proportions could not be calculated. Also in Malawi [26], an increase in malaria after delivery was noticed, from 289/1494 (19\%) at delivery to $1430 / 3864(37 \%)$, at 2 months after delivery, $\mathrm{p}<$ 0.001. In Gabon [24] malaria data during pregnancy were not available and a comparison with postpartum data could not be made.

In Mozambique [23], the IPTp arm showed a decline in malaria proportions from $35 / 493(7 \%)$ at delivery to $13 / 416(3 \%)$ at two months after delivery, $\mathrm{p}=0.004$. By PCR method [25], the proportion of infected women at delivery was $38 / 187$ (20\%) compared to $27 / 138$ (20\%) at 8 weeks postpartum, $\mathrm{p}=0.866$.

\section{Plasmodium falciparum genotyping and sensitive detection}

In Gabon [24], samples with PCR positive placental infections for the merozoite surface antigen-2 (msa2) 
[28] were compared to postpartum samples: five of the 16 puerperal malaria cases $(31 \%$, 95\% CI $11-59 \%)$ carried the same falciparum genotype as detected in the placenta. In Mozambique [25], at eight weeks postpartum $50 \%(13 / 26)$ of women were infected with at least one parasite strain that was present at delivery, and detected with merozoite surface proteins ( $m s p 1$ and $m s p 2)$ [29]. In this study the prevalence of submicroscopic infections in the placebo and IPTp arm combined was 21\% (73/ $352)$ at delivery and $13 \%$ (43/340) postpartum [25].

\section{Parasite density and symptoms}

Five studies (four without IPTp or chemoprophylaxis [18-20,22], and one with chemoprophylaxis [26]) provided information about parasite densities. All reported lower densities during the postpartum period compared to pregnancy, two of which were significantly lower (both $\mathrm{p}=0.02$ ) $[19,20]$.

Four studies provided information about symptoms. In the study from Senegal [19], all episodes that occurred during pregnancy, early postpartum (first 60 days) and in the control period were mild and rapidly cured. Few symptomatic postpartum malaria infections (defined as parasitaemia with a fever) were reported in the study from Mozambique (5.1\%, (2/39) [23] and Cameroon (0/ 33) [20]. In Gabon [24], 61\% (11/18) of malaria episodes in the postpartum group were symptomatic, versus $17 \%$ $(1 / 6)$ in the control group. No study reported severe malaria in the postpartum period.

\section{Discussion}

Nine of 10 reviewed studies suggested that the risk for malaria infection during pregnancy decreased after delivery, and one study suggests an equivalent risk after delivery compared to pregnancy. Postpartum women had more episodes of malaria than non-pregnant, nonpostpartum controls in the three studies that included a control group.

However, the results of this review must be carefully interpreted, as the majority of studies were not designed to document specifically postpartum malaria, and there was a large variability in study designs and outcomes. The use of preventive drugs is an example of such a complicating factor: in more than half $(6 / 11)$ of the studies IPTp or chemoprophylaxis were used during pregnancy. Chloroquine (weekly chemoprophylaxis) and sulphadoxine-pyrimethamine (IPTp) have a long half life and women are protected from malaria until the drug concentration falls below the minimum inhibitory concentration [30]. Depending on the timing of the last dose in pregnancy, drug half life and level of drug resistance, protection could be prolonged into the early postpartum period and may result in an underestimation of the postpartum susceptibility to malaria when compared to non pregnant non postpartum controls who did not use any prevention. In Mozambique, reduced prevalence of malaria parasitaemia at delivery and eight weeks postpartum (detected by malaria smear) was attributed to IPTp-sulphadoxine-pyrimethamine, though the last dose of IPTp was given at a mean time of 77 days before delivery [23]. This is much longer than the one-month estimated protective effect of sulphadoxine-pyrimethamine $[21,30]$. However, when PCR detection was used in a selection of women from the same study [25], it was reported that women in the IPTp group were indeed protected from malaria at delivery, but not anymore at eight weeks postpartum. Many post partum women harboured sub microscopic levels of parasites. Another effect of preventive anti-malarials during pregnancy was seen with use of weekly chemoprophylaxis. In 1985 when P.vivax was still sensitive to chloroquine in PNG, an increase in malaria incidence ( $P$. falciparum, but especially $P$. vivax) was noticed in all gravidae as soon as the chloroquine chemoprophylaxis was stopped at delivery [17]. Such a "rebound effect" was noticed in a chemoprophylaxis study from Tanzania [26] as well as in children [31]. Chemoprophylaxis may have suppressed parasitaemia in pregnancy to sub-microscopic levels, but when it was discontinued at delivery, the parasites became microscopically detectable. This is in agreement with the recent studies, which were able to use PCR genotyping for detection of placental and postpartum parasites $[24,25]$.

In the reviewed studies, postpartum women had more malaria episodes than non-pregnant, non-postpartum controls making plausible the existence of a transition phase, in which the susceptibility returned to pre-pregnancy levels. From the data available, the length of such a period could not be determined. In Senegal, the increased incidence went back to pre-pregnancy levels within 90 days after delivery [19], but in Tanzania women still had an increased prevalence compared to controls six months after delivery [22]. A mechanism to explain the decreased susceptibility in the post-partum period is maternal immunity. Fievet and colleagues observed an impairment in the IL-2 response, and unaffected or enhanced responses in IL-4 and interferongamma, in 33 women from Cameroon followed during their first pregnancy and at 6 months after delivery [20]. This was thought to favor a general depression of cellular immunity in pregnancy, rather than a specific malaria phenomenon. The transition phase from suppressed pregnancy immunity to normal pre-pregnancy levels has also been hypothesized by others [24]. Another mechanism to explain a transition phase is the persistence of malaria parasites from pregnancy due to inadequate treatment. Resistance of $P$. falciparum to chloroquine or sulphadoxine-pyrimethamine was 
mentioned in $55 \%(6 / 11)$ of articles. Ineffective treatments do not radically cure malaria infections [32], and these infections may recrudesce in the postpartum period. The only available P.falciparum genotyping data demonstrated that $30-50 \%$ of the postpartum infections were persistent from delivery until the postpartum period $[24,25]$. Hence, highly effective treatment during pregnancy, or IPTp regimens with highly effective drugs would impact significantly on reducing postpartum infection as well. In addition primiparous women have less antibodies to the CSA binding parasites that cause placental malaria [4] This could explain the increased risk of post partum malaria in primiparous women compared to multiparous mothers observed in six of seven studies reviewed.

To protect the post partum mother and her neonate [33] from malaria, an additional treatment dose of highly effective treatment at delivery may be considered as part of the IPTp strategy. Self-treatment may affect the presence of parasitaemia [34], but this was only reported in two manuscripts. Self-treatment effects are likely to vary with the quality of the drug, the dose and duration of treatment $[35,36]$.

A limitation of this review is that in most published articles microscopy was used to detect malaria, whereas microscopy failed to detect $75 \%$ of the PCR detected parasitaemia during the postpartum period in a recent study [25]. The same study reports that 20 (77\%) out of the 26 women with positive placenta-paired samples had not received treatment during labour because parasitaemia was sub-microscopic. The authors concluded that such untreated sub-microscopic infections persist until the postpartum period and reported a five-fold higher risk (95\% CI 2.49-10.63) of $P$. falciparum infection eight weeks after delivery in women with sub-microscopic placental malaria. This relation between placental and postpartum malaria was not shown in, for example, the study from Senegal [19], where microscopy was used. However, at the same time, in the same area, but in the general population, high levels of sub-patent $P$. falciparum levels (two third of microscopically negative slides) were reported, using PCR [37]. Clearly, more investigation is needed to determine the burden of submicroscopic malaria infections around delivery and in the post partum period.

There was an increased number of symptomatic infections in the postpartum period in Senegal [19] and Gabon [24]. Asymptomatic untreated (sub-microscopic) infections in pregnancy may have become symptomatic after delivery, or asymptomatic episodes were detected and reported as symptomatic due to accompanying fever, as women in the postpartum period are prone to febrile co-morbidities such as mastitis and endometritis. In both studies, the incidence of asymptomatic malaria episodes did not increase as much as for symptomatic episodes.

Data on non-falciparum species and the risk of postpartum infection has only been described for PNG [17]. At least for $P$. vivax and $P$. ovale it would be very useful to know more about the postpartum risks for infection as primaquine, the only available drug for eradication of the liver stage of these species, cannot be used during pregnancy. Postpartum detection may provide a window of opportunity for primaquine therapy so that these species do not relapse in subsequent pregnancies.

\section{Conclusions}

Nine of 10 studies demonstrated a decreasing risk of malaria after pregnancy, but some suggested a risk higher than non-pregnant, non-postpartum controls. A transition phase in which the susceptibility returns to pre-pregnancy levels seems plausible, but from the data available the length of this period could not be determined. Due to methodological differences in the currently available literature the results of this review have to be carefully interpreted. Future studies that precisely document the epidemiology and treatment of malaria episodes during pregnancy, drug resistance, timing of IPTp doses, and self-treatment are needed. Recommendations for the design of a postpartum malaria study are given in Additional file 4.

Sensitive detection for submicroscopic and genotyping of parasites during pregnancy and post partum would be an important component of future studies. The limited data available implies that efforts to detect and treat malaria effectively during pregnancy [38] will prevent women from entering the postpartum period with residual parasitaemia. Administration of an additional treatment dose of an effective drug at delivery to all pregnant women in endemic areas may provide some health benefits to the mother (less anaemia), although be of no direct consequence for the newborn, except in reducing the risk of congenital malaria [33]. Health workers need to be mindful that malaria acquired during pregnancy can have consequences in the postpartum period.

\section{Additional material}

Additional file 1: Table S1. Characteristics of the studies included in the review on postpartum malaria.

Additional file 2: Table S2. Quality measures of the included studies.

Additional file 3: Summary of the pertinent points relating to postpartum susceptibility from 11 manuscripts in alphabetic order of the first author.

Additional file 4: Recommendations for the design of a postpartum malaria study. 


\section{Acknowledgements}

We would like to thank authors of the reviewed studies for the data they shared. We would also like to thank Annemieke van Eijk for providing full text articles. SMRU is supported by The Wellcome Trust of Great Britain, as part of the Oxford Tropical Medicine Research Programme of Wellcome Trust-Mahidol University.

\section{Author details}

'Shoklo Malaria Research Unit, PO Box 46, 63110, Mae Sot Tak, Thailand. ${ }^{2}$ Academic Medical Center, Meibergdreef 9, Postbus 22660, 1100 DD Amsterdam, The Netherlands. ${ }^{3}$ Liverpool School of Tropical Medicine, Pembroke Place, Liverpool, Merseyside L3 5QA, UK. ${ }^{4}$ Faculty of Tropical Medicine, Mahidol University, 420/6 Ratchawithi Road, Bangkok 10400, Thailand. ${ }^{5}$ Centre for Tropical Medicine, Nuffield Department of Clinical Medicine, University of Oxford, CCVTM, Oxford OX3 7LJ, UK.

\section{Authors' contributions}

MB searched for and identified the included studies, extracted data on an Excel spreadsheet and drafted the manuscript. MR searched for and included the studies and helped to draft the manuscript. BB searched for articles, provided raw data, and helped to draft the manuscript. RM did the eligibility assessment and extracted data on an Excel spreadsheet and helped to draft the manuscript. FN did the eligibility assessment and helped to draft the manuscript. All authors read and approved the final manuscript.

\section{Competing interests}

The authors declare that they have no competing interests.

Received: 21 December 2011 Accepted: 13 April 2012

Published: 13 April 2012

\section{References}

1. Wickramasuriya GAW: Some observations on malaria occurring in association with pregnancy. J Obstet Gynaecol 1935, 42:816-834.

2. Desai M, ter Kuile FO, Nosten F, McGready R, Asamoa K, Brabin B, Newman RD: Epidemiology and burden of malaria in pregnancy. Lancet Infect Dis 2007, 7:93-104.

3. McGready R, Lee S, Wiladphaingern J, Ashley E, Rijken M, Boel M, Simpson J, Paw M, Pimanpanarak M, Mu O, Singhasivanon P, White N, Nosten F: Adverse effects of falciparum and vivax malaria and the safety of antimalarial treatment in early pregnancy: a population-based study. Lancet Infect Dis 2011.

4. Fried M, Nosten F, Brockman A, Brabin BJ, Duffy PE: Maternal antibodies block malaria. Nature 1998, 395:851-852.

5. WHO: A Strategic Framework for Malaria Control During Pregnancy in the African Region 2011 [http://www.rollbackmalaria.org/toolbox/ tool_MalariaPreventionlnPregnancy.html].

6. Vallely L, Ahmed Y, Murray SF: Postpartum maternal morbidity requiring hospital admission in Lusaka. Zambia - a descriptive study. BMC Pregnancy Childbirth 2005, 5:1.

7. Barnett S, Nair N, Tripathy P, Borghi J, Rath S, Costello A: A prospective key informant surveillance system to measure maternal mortality - findings from indigenous populations in Jharkhand and Orissa. India BMC Pregnancy Childbirth 2008, 8:6.

8. Nguyen-Dinh P, Steketee RW, Greenberg AE, Wirima JJ, Mulenda O, Williams SB: Rapid spontaneous postpartum clearance of Plasmodium falciparum parasitaemia in African women. Lancet 1988, 2:751-752.

9. Bottero J, Briand V, Agbowai C, Doritchamou J, Massougbodji A, Cot M: Spontaneous postpartum clearance of Plasmodium falciparum parasitemia in pregnant women, Benin. Am J Trop Med Hyg 2011, 84:267-269.

10. Msyamboza KP, Savage EJ, Kazembe PN, Gies S, Kalanda G, D'Alessandro U, Brabin BJ: Community-based distribution of sulfadoxine-pyrimethamine for intermittent preventive treatment of malaria during pregnancy improved coverage but reduced antenatal attendance in southern Malawi. Trop Med Int Health 2009, 14:183-189.

11. Lindsay S, Ansell J, Selman C, Cox V, Hamilton K, Walraven G: Effect of pregnancy on exposure to malaria mosquitoes. Lancet 1972, 2000:355.

12. Riley EM, Schneider G, Sambou I, Greenwood BM: Suppression of cellmediated immune responses to malaria antigens in pregnant Gambian women. Am J Trop Med Hyg 1989, 40:141-144.
13. Sholapurkar SL, Mahajan RC, Gupta AN, Wangoo A: Cellular immunity in pregnant and non-pregnant women with malarial infection. Asia Oceania J Obstet Gynaecol 1990, 16:27-32.

14. WHO:World Health Organization: Postpartum care of mother and newborn: a practical guide Geneva 1998.

15. Malaria in Pregnancy Consortium. [http://www.mip-consortium.org].

16. Liberati A, Altman DG, Tetzlaff J, Mulrow C, Gotzsche PC, loannidis JP, Clarke M, Devereaux PJ, Kleijnen J, Moher D: The PRISMA statement for reporting systematic reviews and meta-analyses of studies that evaluate healthcare interventions: explanation and elaboration. BMJ 2009, 339: b2700.

17. Brabin BJ, Ginny M, Alpers M, Brabin L, Eggelte T, Van der Kaay HJ: Failure of chloroquine prophylaxis for falciparum malaria in pregnant women in Madang, Papua New Guinea. Ann Trop Med Parasitol 1990, 84:1-9.

18. Bray RS, Anderson MJ: Falciparum malaria and pregnancy. Trans $R$ Soc Trop Med Hyg 1979, 73:427-431.

19. Diagne N, Rogier C, Sokhna CS, Tall A, Fontenille D, Roussilhon C, Spiegel A, Trape JF: Increased susceptibility to malaria during the early postpartum period. N Engl J Med 2000, 343:598-603.

20. Fievet N, Cot M, Ringwald P, Bickii J, Dubois B, Le Hesran JY, Migot F, Deloron P: Immune response to Plasmodium falciparum antigens in Cameroonian primigravidae: evolution after delivery and during second pregnancy. Clin Exp Immunol 1997, 107:462-467.

21. Green MD, van Eijk AM, van Ter Kuile FO, Ayisi JG, Parise ME, Kager PA, Nahlen BL, Steketee R, Nettey H: Pharmacokinetics of sulfadoxinepyrimethamine in HIV-infected and uninfected pregnant women in Western Kenya. J Infect Dis 2007, 196:1403-1408.

22. Kortmann HF: Malaria and Pregnancy Amsterdam: University of Amsterdam; 1972.

23. Menendez C, Bardaji A, Sigauque B, Romagosa C, Sanz S, Serra-Casas E, Macete E, Berenquera A, David C, Dobano C, Naniche D, Mayor A, Ordi J, Mandomando I, Aponte JJ, Mabunda S, Alonso PL: A randomized placebocontrolled trial of intermittent preventive treatment in pregnant women in the context of insecticide treated nets delivered through the antenatal clinic. PLoS One 2008, 3:e1934.

24. Ramharter M, Grobusch MP, Kiessling G, Adegnika AA, Moller U, Agnandji ST, Kramer M, Schwarz N, Kun JF, Oyakhirome S, Issifou S, Borrmann S, Lell B, Mordmuller B, Kremsner PG: Clinical and parasitological characteristics of puerperal malaria. J Infect Dis 2005, 191:1005-1009.

25. Serra-Casas E, Menendez C, Dobano C, Bardaji A, Quinto L, Ordi J, Sigauque B, Cistero P, Mandomando I, Alonso PL, Mayor A: Persistence of Plasmodium falciparum parasites in infected pregnant Mozambican women after delivery. Infect Immun 2011, 79:298-304.

26. Steketee RW, Wirima JJ, Bloland PB, Chilima B, Mermin JH, Chitsulo L, Breman JG: Impairment of a pregnant woman's acquired ability to limit Plasmodium falciparu by infection with human immunodeficiency virus type-1. Am J Trop Med Hyg 1996, 55:42-49.

27. Watkinson $\mathrm{M}$, Rushton DI: Plasmodial pigmentation of placenta and outcome of pregnancy in West African mothers. Br Med I (Clin Res Ed) 1983, 287:251-254

28. Ntoumi F, Contamin H, Rogier C, Bonnefoy S, Trape JF, MercereauPuijalon O: Age-dependent carriage of multiple Plasmodium falciparum merozoite surface antigen-2 alleles in asymptomatic malaria infections. Am J Trop Med Hyg 1995, 52:81-88.

29. Mayor A, Serra-Casas E, Bardaji A, Sanz S, Puyol L, Cistero P, Sigauque B, Mandomando I, Aponte JJ, Alonso PL, Menendez C: Sub-microscopic infections and long-term recrudescence of Plasmodium falciparu in Mozambican pregnant women. Malar J 2009, 8:9.

30. White NJ: Intermittent presumptive treatment for malaria. PLoS Med 2005, 2:e3.

31. Greenwood BM, David PH, Otoo-Forbes LN, Allen SJ, Alonso PL: Armstrong Schellenberg JR, Byass P, Hurwitz M, Menon A, Snow RW: Mortality and morbidity from malaria after stopping malaria chemoprophylaxis. Trans $R$ Soc Trop Med Hyg 1995, 89:629-633.

32. McGready R, White NJ, Nosten F: Parasitological efficacy of antimalarials in the treatment and prevention of falciparum malaria in pregnancy 1998 to 2009: a systematic review. BJOG 2011, 118:123-135.

33. Poespoprodjo JR, Fobia W, Kenangalem E, Hasanuddin A, Sugiarto $P$, Tjitra E, Anstey NM, Price RN: Highly effective therapy for maternal malaria associated with a lower risk of vertical transmission. J Infect Dis 2011, 204:1613-1619 
34. Hodel EM, Kabanywanyi AM, Malila A, Zanolari B, Mercier T, Beck HP, Buclin T, Olliaro P, Decosterd LA, Genton B: Residual antimalarials in malaria patients from Tanzania-implications on drug efficacy assessment and spread of parasite resistance. PLoS One 2009, 4:e8184.

35. Atemnkeng MA, De Cock K, Plaizier-Vercammen J: Quality control of active ingredients in artemisinin-derivative antimalarials within Kenya and DR Congo. Trop Med Int Health 2007, 12:68-74.

36. Newton PN, McGready R, Fernandez F, Green MD, Sunjio M, Bruneton C, Phanouvong S, Millet P, Whitty CJ, Talisuna AO, Proux S, Christophel EM, Malenga G, Singhasivanon P, Bojang K, Kaur H, Palmer K, Day NP, Greenwood BM, Nosten F, White NJ: Manslaughter by fake artesunate in Asia-will Africa be next? PLoS Med 2006, 3:e197.

37. Bottius E, Guanzirolli A, Trape JF, Rogier C, Konate L, Druilhe P: Malaria: even more chronic in nature than previously thought; evidence for subpatent parasitaemia detectable by the polymerase chain reaction. Trans R Soc Trop Med Hyg 1996, 90:15-19.

38. WHO: Guidelines for the treatment of malaria. 2 edition. Geneva: WHO; 2010.

doi:10.1186/1475-2875-11-114

Cite this article as: Boel et al:: The epidemiology of postpartum malaria: a systematic review. Malaria Journal 2012 11:114.

\section{Submit your next manuscript to BioMed Central} and take full advantage of:

- Convenient online submission

- Thorough peer review

- No space constraints or color figure charges

- Immediate publication on acceptance

- Inclusion in PubMed, CAS, Scopus and Google Scholar

- Research which is freely available for redistribution

Submit your manuscript at www.biomedcentral.com/submit 